\title{
Novel MYH11 and ACTA2 mutations reveal a role for enhanced TGF $\beta$ signaling in
}

\section{FTAAD}

Marjolijn Renard (MSc) ${ }^{1, *+\ddagger}$, Bert Callewaert (MD, PhD) ${ }^{1, \ddagger}$, Machteld Baetens (IE) ${ }^{1}$, Laurence Campens (MD) ${ }^{1}$, Kay MacDermot (MD) ${ }^{2}$, Jean-Pierre Fryns (Prof, MD, PhD) ${ }^{3}$, Maryse Bonduelle (Prof, MD) ${ }^{4}$, Hal Dietz (Prof, MD) ${ }^{5,6}$, Isabel Mendes Gaspar (MD) ${ }^{7}$, Diogo Cavaco $(\mathrm{MD})^{7}$, Eva-Lena Stattin (MD, PhD) ${ }^{8}$, Constance Schrander-Stumpel (MD) ${ }^{9}$, Paul Coucke (Prof, PhD, IE) $)^{1}$, Bart Loeys (Prof, MD, PhD) $)^{1,10}$, Anne De Paepe (Prof, MD, PhD) $)^{1}$, Julie De Backer $(\mathrm{MD}, \mathrm{PhD})^{1}$

${ }^{1}$ Center for Medical Genetics, University Hospital of Ghent, Ghent, Belgium, ${ }^{2}$ North West Thames Regional Genetics Service, Northwick Park \& St. Mark's hospital, Middlesex, UK, ${ }^{3}$ Center for Medical Genetics, University Hospital of Leuven, Leuven, Belgium, ${ }^{4}$ Center for Medical Genetics, University Hospital of Brussels, Brussels, Belgium, ${ }^{5}$ McKusick-Nathans Institute of Genetic Medicine, Johns Hopkins University School of Medicine, Baltimore, MD, USA, ${ }^{6}$ Howard Hughes Medical Institute, Johns Hopkins University School of Medicine, Baltimore, MD, USA, ${ }^{7}$ Dept. de Genetica Medica, Hospital Egas Moniz and Cardiology Department, Hospital Santa Cruz, CHLO, Lisboa, Portugal, ${ }^{8}$ Department of Medical Biosciences, Medical and Clinical Genetics, Umeå University, Sweden, ${ }^{9}$ Department of Clinical Genetics and Research Institute Growth and Development (GROW), Maastricht University Medical Center $\mathrm{UMC}^{+}$, Maastricht, The Netherlands, ${ }^{10}$ Center for Medical Genetics, University Hospital of Antwerp, Antwerp, Belgium.

* To whom correspondence should be addressed at: Marjolijn Renard, Center for Medical Genetics, De Pintelaan 185, B-9000 Ghent, Belgium, phone 0032-9-332.55.35, fax 0032-9332.65.49, e-mail Marjolijn.Renard@ugent.be

$\$$ These authors contributed equally to this work 
This study was supported in part by the Fund for Scientific Research, Flanders (Belgium) [G.0094.06]; Fighting Aneurysmal Disease [EC-FP7]; the Special Research Fund of the Ghent University [BOF10/GOA/005]; and a Methusalem grant from the flemmisch government and the Ghent University to ADP [08/01M01108]. B. Loeys, J. De Backer, B. Callewaert and M. Renard are/were, respectively, senior clinical investigators (BL and JDB), postdoctoral fellow (BC) and junior scientific investigator (MR) supported by the Fund for Scientific Research, Flanders (Belgium). 


\section{ABSTRACT}

Background Thoracic aortic aneurysm / dissection (TAAD) is a common phenotype that may occur as an isolated manifestation or within the constellation of a defined syndrome. In contrast to syndromic TAAD, the elucidation of the genetic basis of isolated TAAD has only recently started. To date, defects have been found in genes encoding extracellular matrix proteins (fibrillin-1, FBN1; collagen type III alpha 1, COL3A1), proteins involved in transforming growth factor beta (TGF $\beta$ ) signaling (TGF $\beta$ receptor 1 and 2, TGFBR1/2; and $S M A D 3)$ or proteins that build up the contractile apparatus of aortic smooth muscle cells (myosin heavy chain 11, MYH11; smooth muscle actin alpha 2, ACTA2; and MYLK).

Methods and results In 110 non-syndromic TAAD patients that previously tested negative for FBN1 or TGFBR1/2 mutations, we identified 7 ACTA2 mutations in a cohort of 43 familial TAAD patients, including 2 premature truncating mutations. Sequencing of MYH11 revealed an in frame splice-site alteration in one out of two probands with TAA(D) associated with PDA but none in the series of 22 probands from the cohort of 110 patients with nonsyndromic TAAD. Interestingly, immunohistochemical staining of aortic biopsies of a patient and a family member with MYH11 and patients with ACTA2 missense mutations showed upregulation of the TGF $\beta$ signaling pathway.

Conclusions MYH11 mutations are rare and typically identified in patients with TAAD associated with PDA. ACTA2 mutations were identified in $16 \%$ of a cohort presenting familial TAAD. Different molecular defects in TAAD may account for a different pathogenic mechanism of enhanced TGF $\beta$ signaling.

Keywords: thoracic aortic aneurysm, myosin heavy chain 11 , smooth muscle $\alpha$-actin, TGF $\beta$ signaling. 


\section{Introduction}

Aortic aneurysms and dissections are a frequent cause of morbidity and mortality in the Western world $[1,2]$. In thoracic aortic aneurysms and dissections (TAAD), the dilatation most commonly involves the aortic root and/or ascending aorta. Dissections are mostly of the Stanford type A, involving the ascending aorta, Stanford type B dissections (occurring distally from the subclavian artery) are less common. TAAD may occur within the context of defined monogenic syndromes, in particular the Marfan syndrome (MFS). More commonly, TAAD presents in the absence of a clear syndromic constellation, i.e. non-syndromic TAAD. In up to $20 \%$ of TAAD patients a positive family history is observed, clearly indicating a genetic contribution in the etiology [3].

The search for the underlying molecular defect in non-syndromic TAAD has confirmed the genetic heterogeneity of the syndrome and has increased our insights into the complex pathophysiology of aneurysm formation. Occasionally mutations in FBN1 and TGFBR1/2 have been documented [4] in patients presenting either mild connective tissue manifestations or widespread vascular disease. As such, these patients may represent variable expression of MFS or Loeys-Dietz syndrome (LDS). Three loci for TAAD have been mapped, but the causal gene has not been identified yet. One locus was mapped to 5q13-14 in nine out of fifteen families with autosomal dominantly segregating TAAD with reduced penetrance [5]; a second locus on 11q23-24 was identified in a single, large family characterized by a diffuse vascular disease, designated as FAA [6]. The third locus was recently identified by Guo and colleagues, the locus, TAAD5, was mapped to chromosome 12 $(19,5-68,1 \mathrm{Mb})[7]$. The family in which this locus was identified is characterized by stable ascending aortic aneurysm with a low risk of dissection and a marked decreased penetrance in females. 
Four novel gene defects have been reported in the context of TAAD. First, mutations in myosin heavy chain protein $11(M Y H 11)$, a specific contractile protein of smooth muscle cells, have been identified in 2 families with dominant transmission of TAAD in conjunction with patent ductus arteriosus (PDA) [8]. In another study, in which 2 additional MYH11 mutations were found, it was shown that a structural defect in MYH11 disturbs contractile smooth muscle cell function and leads to lower aortic compliance and elastolysis as well as focal vascular smooth muscle hyperplasia [9]. Second, mutations in ACTA2 (encoding the smooth muscle cytoskeletal protein actin $\alpha 2$ ) were identified in families with aortic root dilatation and a variety of associated manifestations, including patent ductus arteriosus (PDA), bicuspid aortic valve (BAV), iris flocculi, livedo reticularis, cerebrovascular accident (CVA) and stenosis of the aortic vasa vasorum [10]. In addition TAAD caused by ACTA2 mutations is occasionally combined with premature onset of coronary artery disease, stroke and Moya-Moya disease [11]. Recently, 5 new papers were published, all identifying new and/or previously published ACTA2 mutations in TAAD patients with a variable association of extravascular traits [12-16]. These findings make this gene defect the most common cause of familial TAAD identified to date. Third, mutations were recently identified in the gene encoding myosin light chain kinase $(M Y L K)$, a kinase that is involved in smooth muscle cell contractility. The mutations found so far have an effect on kinase activity or calmodulin binding properties of the protein, clinically leading to acute aortic dissection with minimal or no aortic enlargement prior to dissection [17]. The most recently identified gene in the context of TAAD is the SMAD3 gene, which is a member of the TGF $\beta$ pathway that is essential for TGF $\beta$ signal transmission. Patients harboring SMAD3 mutations display a phenotype mainly characterized by ascending aortic aneurysm and dissection in association with early onset osteoarthritis [18]. 
The identification of MYH11, ACTA2 and MYLK gene mutations confirm the importance of the smooth muscle cell contractile apparatus in maintaining the structural integrity of the ascending aorta, but the precise pathophysiological mechanism underlying the development of aneurysms remains unclear [10].

We screened a large cohort of TAAD patients in whom FBN1 and TGFBR1/2 were excluded, for both ACTA2 and MYH11 to further delineate the associated phenotypes. We also assessed the involvement of the TGF $\beta$ pathway using dedicated staining methods. We identified one novel MYH11 mutation, and 5 novel and 2 previously identified ACTA2 mutations and provide evidence that enhanced TGF $\beta$ signaling contributes to the pathophysiology of aneurysms associated with MYH11 mutations and ACTA2 missense mutations.

\section{Material and methods}

\subsection{Patients and samples}

The cohort consisted of 110 probands from Europe and the United States of America. All probands were evaluated by a clinical geneticist at the referring center. Clinical information was obtained by means of a detailed checklist. All patients screened had an ascending aortic aneurysm (Z-score > 3) either at the sinuses of Valsalva or the more distal part of the ascending aorta, and neither fulfilled the diagnostic criteria for MFS [19] or presented with a syndromic form of TAAD. All patients tested negative for FBN1 and TGFBR1/2 by sequencing. Family history of TAAD was present in 43 of the 110 probands. All 110 probands were screened for ACTA2 mutations and a randomly limited set of 22 probands was screened for MYH11. In addition, we screened the MYH11 gene in two patients associating TAAD associated with PDA, who were not included in the cohort of 110 probands. Mutation-positive families were clinically re-evaluated by the referring clinician. 
Genomic DNA was extracted from peripheral blood and/or cultured skin fibroblasts. Skin fibroblasts were cultured in Dulbecco's modified Eagle's Medium (DMEM) supplemented with $10 \%$ fetal bovine serum (FBS) in the presence of antibiotics and antimycotics. Aortic specimens were obtained at the occasion of aortic root replacement. Aortic tissues were available from family 1 member II:3, family 2 member III:3, family 4 member II:4, and family 8 member III:1 and III:6 (Figure 2). Aortic tissue from control patients who died of unrelated causes was obtained during autopsy. This study was approved by the ethics committee of the Ghent University Hospital and all referring centers, and an appropriate informed consent was obtained from all patients involved in the study.

\subsection{Molecular analysis}

Genomic DNA was amplified by touchdown PCR of all coding exons with their exonintron boundaries of ACTA2 and MYH11, respectively, using forward and reverse primers located in the flanking introns (primer sequences available upon request). The PCR products were analyzed by gel electrophoresis and visualized by ethidium bromide staining on $2 \%$ agarose gels. All amplimers were directly sequenced on the ABI PRISM 3730 automated sequencer (Applied Biosystems) using the BigDye terminator cycle sequencing chemistry. These sequences were compared to the wild-type sequence as submitted to GenBank Accession number NM_022844.2 and NM_002474 of MYH11 and ACTA2, respectively. All mutations were confirmed to be segregating with the phenotype and found absent in a panel of 100 normal control individuals.

\subsection{Staining of aortic sections}

Formalin-fixed and paraffin-embedded aortic tissue was cut in $5 \mu \mathrm{m}$ sections and prepared for immunohistochemical analysis. Sections were stained for phospho-Smad2 (Cell 
Signaling Technologies) and CTGF (Abcam). Briefly, antigens were unmasked using 1mM EDTA pH 8 and auto-peroxidase activity was inhibited. Sections were blocked with normal goat serum (Vectastain) and incubated with antibodies directed against CTGF and pSmad2 (Ser465/467). Subsequently, sections were incubated with a secondary antibody goat-antirabbit IgG (Vectastain), ABC (Avidin: Biotinylated enzyme Complex) reagent (Vectastain) and $\mathrm{DAB}$ (3,3'-Diaminobenzidine) peroxidase (Vectastain). Sections were dehydrated in xylene and mounted. Additionally, immunofluorescent co-staining for fibronectin-1 (FN1) and smooth muscle $\alpha$-actin was performed. Sections were deparaffinized, rehydrated, blocked with 5\% bovine serum albumin (BSA, Sigma Aldrich), and subsequently incubated with primary antibodies directed against FN1 (Abcam) and smooth muscle $\alpha$-actin (Sigma Aldrich). Next, the sections were incubated with secondary antibodies, donkey anti-rabbit alexa 488 (Invitrogen) and goat anti-mouse Cy3 (GE Healthcare), respectively. Finally, the stained tissues were covered with Vectashield dapi (Vector Laboratories) and cover-slipped. 


\section{Results}

\subsection{Genomic sequencing of MYH11 and ACTA2}

In 110 consecutive patients with TAAD, of which 43 familial cases and 67 isolated cases, ACTA2 mutations were identified in 7 probands, all with a positive family history of TAAD. In family 1 and 2 we identified mutations that result in a premature termination codon: c.940C>T; p.Arg314X (family 1) and c.1019_1020delCT; p.Ser340Cys fs X25 (family 2). In families 3-7, five ACTA2 missense substitutions (c.124C>A; p.His42Asn, c.115C > T; p.Arg39Cys, c.145G>A; p.Met49Val, c.112G>A; p.Gly38Arg, and c.182A>G; p.Gln61Arg, respectively) were identified, of which 3 are novel (p.His42Asn, p.Gly38Arg, and p.Gln61Arg) and the latter two were previously reported (p.Arg39Cys and p.Met49Val) [13]. See Figure 1. No ACTA2 mutations were found in the 67 sporadic TAAD patients.

In 2 families with TAAD associated with PDA, a causal aberration in MYH11 was found in one family (family 8): IVS32+1G>A mutation in intron 32 (Figure 1). In contrast, no MYH11 mutation was found in a series of 22 patients with non-syndromic FTAAD.

\subsection{Clinical phenotypes}

Family 1

The 70-year-old female proband presented at the emergency department with acute type A aortic dissection and died during surgery. Autopsy revealed no other dysmorphic features. The type A dissection originated in an aortic root aneurysm of $50 \mathrm{~mm}$ and stretched down into the descending aorta. Section of the coronary arteries revealed a distal stenosis of $75 \%$ of the left anterior descending artery. The proband's mother had died suddenly at the age of 63 and the proband's father had a fatal cerebrovascular accident at age 77 . The proband's brother underwent aortic root replacement at the age of 45 . At the age of 65 , he required 
revision because of partial detachment of the distal suture between the prosthesis and the aorta with formation of a pseudoaneurysm. Because of this family history, the three children of the proband (respectively aged 48, 47 and 45 years) were referred to the genetics department for further assessment. Both daughters presented normal echocardiographic findings, whereas the 47-year-old son presented mild dilatation of the aortic root at the sinuses of Valsalva (42 mm, $\mathrm{Z}=2.03$ ). Subsequent screening of his children revealed mild aortic dilatation in the 21-yearold daughter ( $38 \mathrm{~mm}$ at the sinuses of Valsalva, $\mathrm{Z}=2.9$ ). None of the affected family members presented dysmorphic features, connective tissue abnormalities, skin manifestations or other vascular involvement at the time of evaluation.

Family 2

The currently 84-year-old male proband presented to the clinic at the age of 68 years with a type B aortic dissection for which he was successfully stented. He did not present any clinical features reminiscent of MFS or LDS. His parents died at old age and not due to aortic aneurysm. His paternal aunt died in her late thirties during surgery for a ruptured abdominal aneurysm. His 40-year old son has borderline aortic root measurements - the mutation was excluded in him. A paternal cousin of the proband required aortic root replacement for aneurysm formation associated with valvular insufficiency at the age of 56 . He died at age 70 of a ruptured abdominal aortic aneurysm.

\section{Family 3}

The 32-year-old male proband was diagnosed with an aortic root aneurysm and associated aortic valve insufficiency. He did not have any clinical signs of a connective tissue disorder. His mother died suddenly of a type A aortic dissection at the age of 48 . His maternal 
aunt and her 32-year-old son also died from aortic dissection. The family was lost for further follow-up.

Family 4

The 47-year-old male proband is the third child of non-consanguineous parents. At the age of 32, he required an emergency Bentall procedure for a type A aortic dissection. Followup CT-angiography at the age of 36 revealed a dilatation of the aorta distal from the prosthesis with a maximal diameter of $50 \mathrm{~mm}$, a saccular aneurysm of the descending thoracic aorta of $38 \mathrm{~mm}$ (from the level of the left atrium to the diaphragm) with a chronic dissection in the most distal $1.5 \mathrm{~cm}$ and an infrarenal aortic aneurysm of $37 \mathrm{~mm}$, extending to the iliac bifurcation, with a mural thrombus. Arteriography of the coronary and cerebral arteries was normal. The 22-year-old son of the proband showed borderline aortic diameters on echocardiography (36 $\mathrm{mm}$ at the sinuses of Valsalva). Family history was positive for sudden death in the proband's oldest brother at the age of 26 , for which the underlying etiology remained unconfirmed. This brother's son was also affected, but no clinical information was available. The second brother of the proband was admitted to the emergency department at the age of 44 with an acute type A dissection extending to the abdominal aorta. Emergency surgery was successful, but was complicated by an inferior myocardial infarction. Pathology showed myxoid deposits in the intima, but no cystic media necrosis. The three children of this brother are all healthy. The proband's father died at the age of 55 from an internal bleeding of unknown origin. His mother was healthy until old age. None of the patients showed any other connective tissue signs, except for livedo reticularis in the proband.

\section{Family 5}


The proband is a 14-year-old girl, who was referred to the genetics department after her mother's sudden death from an acute type A aortic dissection during her third pregnancy. The proband was known with a patent ductus arteriosus but with normal aortic root measurements (26 $\mathrm{mm}$ at the sinuses of Valsalva; $\mathrm{Z}=0.87$ ). Upon physical examination she presented mild skin translucency and joint hyperlaxity. Routine echocardiographic evaluation of her sister at the age of 6 years revealed mild dilatation at the sinuses of Valsalva $(22 \mathrm{~mm}$, $\mathrm{Z}=2$ ) and a small patent ductus arteriosus.

\section{Family 6}

The female proband was referred at the age of 27 because of a positive family history of aortic disease. Echocardiography showed normal aortic root and ascending aortic diameters but a CT-scan revealed a chronic type B aortic dissection originating at the left subclavian artery and extending to the renal arteries with a maximal diameter of $60 \mathrm{~mm}$ at the descending thoracic aorta. She underwent surgical interposition of a Dacron conduit. Five years after surgery she developed a mild aneurysm at the left subclavian artery $(1.3 \mathrm{~cm})$ and dilatation $(4.3 \mathrm{~cm})$ of the native aorta (false lumen) in the descending aorta. Although her mother was asymptomatic, the proband's maternal aunt had an extensive cardiovascular history. At the age of 20 , she was diagnosed with mitral valve prolapse and ascending aortic dilatation progressing to an aneurysm requiring surgery at the age of 34 . She is currently also under follow-up for an abdominal aortic aneurysm. The proband's niece underwent aortic surgery at age 27 for a dissection of a thoraco-abdominal aneurysm. Her medical history also included profuse postpartum uterine hemorrhage and translucent skin with prominent subcutaneous vessels, easy bruising, early-onset varicose veins, small joint hypermobility, tendon/muscle rupture and atrophic scars, reminiscent of vascular Ehlers-Danlos syndrome. 


\section{Family 7}

The 52-year-old male proband underwent a composite graft procedure because of an ascending aortic aneurysm with valvular insufficiency at the age of 29 . At age 35 , he presented with a dissection of the suprarenal abdominal aorta expanding to the superior mesenteric artery and with occlusion of the left common iliac artery. He is currently under follow-up for an enlarging ascending and arch aortic aneurysm distal from the prothesis. His father and paternal grandfather both died of aortic dissection between the age of 30 and 35, his brother suffered from a type A aortic dissection at age 35 .

\section{Family 8}

The proband is a 36-year-old female, with a history of a PDA that was surgically corrected during childhood. Progressive aortic root dilatation was first noted at age 28 and treated with aortic root replacement at the age of 34 . Clinical evaluation revealed no skeletal, ocular, skin or pulmonary problems. Her father suddenly died at the age of 63 , probably from an aortic dissection. Her eldest sister underwent surgery for a PDA at the age of 12, and died suddenly at the age of 33. Autopsy revealed a type A aortic dissection extending in both carotid arteries and to the iliac bifurcation. Lungs showed minor emphysematous changes. Another sister died shortly after birth, probably due to congestive heart failure secondary to a PDA. Both other sisters are healthy, but the child of one of the sisters has severe pulmonary valve stenosis. The latter child's paternal uncle had coronary artery disease at the age of 60 . His 30-year-old daughter is currently in good general health, but had surgery at the age of 4 for a severe pulmonary valve stenosis. 
All pedigrees are shown in Figure 2. Affected family members are indicated with a black box and the presence or absence of the mutation is indicated with a plus or minus sign, respectively.

\subsection{Immunohistochemistry of the aortic wall in patients with ACTA2 and MYH11 mutations}

The available aortic sections were co-stained with antibodies directed against smooth muscle $\alpha$-actin and fibronectin-1 (FN1). Immunofluorescent FN1 staining showed welldefined, intact FN fibrils and elastic fibers in aortic tissue in the control individuals, while the aortic tissue of MYH11- and ACTA2-mutation positive patients showed a marked fragmentation of the elastic fibers and a defective FN assembly in the tunica media. Smooth muscle $\alpha$-actin staining was increased in $M Y H 11$-mutation positive tissues when compared to controls, indicating hyperproliferation of smooth muscle cells. Actin staining in aortic tissue from ACTA2 mutation-positive patients was variable. The morphology of the smooth muscle cells was also altered: patient aortic tissues from both ACTA2 and MYH11 mutation-positive patients showed the presence of few round shaped cells, while in the control tissues the majority of the smooth muscle cells were elongated (Figure 4).

\subsection{TGF $\beta$ signaling pathway}

In order to investigate alterations in TGFbeta signaling, all available aortic sections of patients and control individuals were stained for connective tissue growth factor (CTGF) and phospho-Smad2 (pSmad2). An increased intensity of staining for both proteins was seen in all family members with a MYHI1 mutation as compared to the control tissues (only 1 family member is shown) (Figure 3). The staining intensity of CTGF and pSmad2 in the ACTA2 mutation-positive patients was variable. Increased staining was seen in only one of the three aortic biopsies of the patients with an ACTA2 mutation, namely in patient II:4 from family 4 
with an ACTA2 missense mutation (only 1 out of 2 stainings of the patients with a premature termination codon is shown) (Figure 3). This finding suggests that upregulation of TGF $\beta$ signaling is involved in $M Y H 11$ pathogenesis and to a lesser extent in ACTA2 pathogenesis. 


\section{Discussion}

Recent insights in the molecular basis of TAAD have shown the causal contribution of both $M Y H 11$ and ACTA2 mutations to the development of aortic aneurysms in at least a subset of patients [8-11]. In the present study, we therefore comprehensively screened both genes in a large cohort of patients with (familial) TAAD. While no MYH11 mutations were found in families with non-syndromic TAAD, we did identify a mutation in one out of two families associating TAAD with PDA. The MYH11 mutation-negative family was also negative for ACTA2. To our knowledge, this is the fifth MYH11 mutation reported to date and confirms that MYH11 mutations are rare and causal to this specific phenotype of TAAD associated with PDA. In contrast, we identified 7 ACTA2 mutations in the large cohort of 110 TAAD probands. A prevalence of $16 \%$ in patients with a positive family history indicates that ACTA2 is mutated in a significant proportion of familial TAAD patients $(7 / 43)$.

At the molecular level, the MYH11 mutation (IVS32+1G>A) affects the canonical splice site sequence and is predicted to result in the loss of exon 32 , and thus an in-frame deletion of 71 amino acids. This causes a conformational change of the $\alpha$-helical coiled coil domain of the smooth muscle myosin heavy chain and impairs the motor function of the protein and its assembly with a homodimeric counterpart. This mutation is similar to the IVS32+1G $>\mathrm{T}$ mutation previously reported and further supports a dominant negative effect for $M Y H 11$ mutations [8].

All missense mutations in ACTA2 reported here affect highly conserved amino acids and result in a deleterious alteration of the $2^{\text {nd }}$ subdomain surface, a domain important for the conformational change of actin upon the exchange of ADP for ATP, in its turn important in filament assembly dynamics [20]. A dominant negative mechanism with reduced assembly and incomplete and disorganized actin filament assembly was previously suggested for ACTA2 mutations [10]. The current study demonstrates for the first time that TAAD may also 
result from premature truncating mutations in ACTA2. However, both nonsense mutations are predicted to escape nonsense mediated decay and could as such still exert a dominant negative effect. This confirms the hypothesis formulated by Guo et al, who suggest that mutations resulting in true null alleles are inherited only with a recessive transmission pattern [10].

Possible limitations to the applied molecular studies are that both genes, ACTA2 and MYH11, were not tested for large deletions or insertions with Multiplex Ligation-dependent Probe Amplification (MLPA). Also, MLPA analysis for FBN1 and TGFBR1/2, was limited to a specific subset of patients with a high clinical suspicion for Marfan or Loeys-Dietz syndrome respectively (defined as patients presenting a TAA in addition to other characteristic clinical features of Marfan or Loeys-Dietz syndrome). Screening of the recently identified TAAD causing genes SMAD3 and MYLK in the remaining cohort is ongoing.

Both ACTA2 and MYH11 mutation-positive families reported so far display highly variable phenotypic expression with manifestations occurring at all age categories and not limited to the cardiovascular system $[9,11,12,16,21]$. The spectrum of clinical manifestations reported in this series is less extensive. More specifically, we did not observe significant manifestations of vasculo-occlusive disease nor of ocular, gastro-intestinal or urinary tract abnormalities, as reported by Milewicz et al [16]. One patient in our series presented livedo reticularis and we confirmed the presence of bicuspid aortic valve and patent ductus arteriosus in the clinical spectrum of the ACTA2 phenotype. There are several explanations possible for these discrepancies: firstly, it is very likely that we had a selection bias towards TAA since recruitment of patients and families occurred at a TAA reference centre (Centre for Medical Genetics, Ghent), secondly patients were clinically examined at different centers and no uniform vascular imaging protocol was used; subclinical vasculo- 
occlusive disease cannot be excluded. Lastly, the relative small number of families described cannot exclude the presence of other clinical manifestations.

Attempts have been made to identify genotype-phenotype correlations in ACTA2 related disease. Guo et al suggested that the p.Arg258His mutation was primarily associated with stroke, PDA and Moya Moya disease, and p.Arg118Gln and p.Arg149Cys mutations were associated with Coronary Artery Disease (CAD) [11], but this has been refuted in subsequent studies $[12,14]$. Supplemental table S1 shows no clear genotype-phenotype correlations in the majority of ACTA2 mutation-positive patients.

Based on these observations regarding genotype-phenotype correlations, it seems likely that ACTA2 mutation related disease is similar to other heritable connective tissue disorders such as Marfan syndrome displaying a broad phenotypic spectrum that cannot be simply explained by the mutation characteristics. This suggests that other (environmental, epigenetic) factors may play a role and definitely merits further research.

The pathogenesis of TAAD caused by MYH11 or ACTA2 mutations remains intriguing but implicates a defective contractile apparatus. We observed a variable and increased smooth muscle $\alpha$-actin staining in ACTA2 mutation-positive patients and $M Y H 11$ mutation-positive patients, respectively. Moreover, aortic tissues of mutation-positive patients were characterized by the presence of a few round shaped, proliferating smooth muscle cells in the aortic media. These smooth muscle cells are thought not to produce contractile proteins in contrast to the elongated cells present in control tissues [22]. Nevertheless, these patients at least those with MYH11 mutation- show an increased smooth muscle $\alpha$-actin staining, this contradictory finding might be explained by hyperproliferation of smooth muscle cells in the media as reported previously [10-12], rather than an increase in staining intensity of a single cell. In patients with an ACTA2 missense mutation as well as with a MYH11 mutation, we 
found direct evidence of increased TGF $\beta$ signaling in aortic tissue sections, as observed in syndromic forms of TAAD including Marfan syndrome, Loeys-Dietz syndrome and FBLN4 and ELN-related cutis laxa [23-26]. This is in contrast with the previously reported experiments of Pannu and colleagues [9] that showed that there was no increased CTGF staining nor increased expression of CTGF and TGF $\beta 1$ in aortic tissue and smooth muscle cells of MYH11 patients. The precise contribution of TGF $\beta$ signaling to the pathogenesis of non-syndromic aortic aneurysms remains to be clarified [27]. Binding and contractility of the cytoskeleton via integrins is required for inducing a conformational change in fibronectin, allowing the association of fibronectin dimers into a stable insoluble fibrillar matrix (for a review see [28]). A stable fibronectin fibrillar matrix is in its turn indispensable for C-terminal association of fibrillin-1 monomers into bead-like structures and their linear assembly into microfibrils [29]. Furthermore, fibronectin microfibrils incorporate the large latent complex (TGF $\beta$ bound to its latency associated peptide, LAP, and latent TGF $\beta$ binding protein, LTBP) and transfer it to fibrillin-1 microfibrils which form the elastic fibers together with elastin [3033] (Figure 5A). Therefore, we hypothesize that increased TGF $\beta$ signaling could be explained by the fact that myosin or actin filaments that can no longer assemble correctly due to the presence of mutations in MYH11 or ACTA2, respectively, will result in a defective fibronectin fibril assembly at the cell surface, as seen in the fluorescent immunohistochemical staining (Figure 4). This in turn will lead to a defective fibrillin-1 assembly into microfibrils and will inhibit the sequestration of latent TGF $\beta$, making it more prone to activation and leading to an increased amount of nuclear phosphorylated Smad2 and CTGF (Figure 5B). Further studies on the role of TGF $\beta$ signaling in ACTA2/MYH11 deficient patients are needed, as they could have a direct impact on the treatment options in these patients, such as the possibility of administrating losartan, a TGF $\beta$ antagonist to counteract the consequences of excess active TGF $\beta$ [34]. 


\section{Acknowledgements}

We thank P. Van Acker for excellent technical assistance with the molecular analyses. The authors of this manuscript have certified that they comply with the Principles of Ethical Publishing in the International Journal of Cardiology [35]. 


\section{References}

[1] Lilienfeld DE, Gunderson PD, Sprafka JM, Vargas C. Epidemiology of aortic aneurysms: I. Mortality trends in the United States, 1951 to 1981. Arteriosclerosis. 1987;7:637-43.

[2] Milewicz DM, Michael K, Fisher N, Coselli JS, Markello T, Biddinger A. Fibrillin-1 (FBN1) mutations in patients with thoracic aortic aneurysms. Circulation. 1996;94:2708-11. [3] Biddinger A, Rocklin M, Coselli J, Milewicz DM. Familial thoracic aortic dilatations and dissections: a case control study. J Vasc Surg. 1997;25:506-11.

[4] Pannu H, Fadulu VT, Chang J, et al. Mutations in transforming growth factor-beta receptor type II cause familial thoracic aortic aneurysms and dissections. Circulation. 2005;112:513-20.

[5] Guo D, Hasham S, Kuang SQ, et al. Familial thoracic aortic aneurysms and dissections: genetic heterogeneity with a major locus mapping to 5q13-14. Circulation. 2001;103:2461-8. [6] Vaughan CJ, Casey M, He J, et al. Identification of a chromosome 11q23.2-q24 locus for familial aortic aneurysm disease, a genetically heterogeneous disorder. Circulation. 2001;103:2469-75.

[7] Guo DC, Regalado ES, Minn C, et al. Familial thoracic aortic aneurysms and dissections: identification of a novel locus for stable aneurysms with a low risk for progression to aortic dissection. Circ Cardiovasc Genet. 2011;4:36-42.

[8] Zhu L, Vranckx R, Khau Van Kien P, et al. Mutations in myosin heavy chain 11 cause a syndrome associating thoracic aortic aneurysm/aortic dissection and patent ductus arteriosus. Nat Genet. 2006;38:343-9.

[9] Pannu H, Tran-Fadulu V, Papke CL, et al. MYH11 mutations result in a distinct vascular pathology driven by insulin-like growth factor 1 and angiotensin II. Human molecular genetics. 2007;16:2453-62. 
[10] Guo DC, Pannu H, Tran-Fadulu V, et al. Mutations in smooth muscle alpha-actin (ACTA2) lead to thoracic aortic aneurysms and dissections. Nature genetics. 2007;39:148893.

[11] Guo DC, Papke CL, Tran-Fadulu V, et al. Mutations in smooth muscle alpha-actin (ACTA2) cause coronary artery disease, stroke, and Moyamoya disease, along with thoracic aortic disease. American journal of human genetics. 2009;84:617-27.

[12] Disabella E, Grasso M, Gambarin FI, et al. Risk of dissection in thoracic aneurysms associated with mutations of smooth muscle alpha-actin 2 (ACTA2). Heart. 2011;97:321-6. [13] Hoffjan S, Waldmuller S, Blankenfeldt W, et al. Three novel mutations in the ACTA2 gene in German patients with thoracic aortic aneurysms and dissections. European journal of human genetics : EJHG. 2011.

[14] Morisaki H, Akutsu K, Ogino H, et al. Mutation of ACTA2 gene as an important cause of familial and nonfamilial nonsyndromatic thoracic aortic aneurysm and/or dissection (TAAD). Human mutation. 2009;30:1406-11.

[15] Yoo EH, Choi SH, Jang SY, et al. Clinical, pathological, and genetic analysis of a Korean family with thoracic aortic aneurysms and dissections carrying a novel Asp26Tyr mutation. Ann Clin Lab Sci. 2010;40:278-84.

[16] Milewicz DM, Ostergaard JR, Ala-Kokko LM, et al. De novo ACTA2 mutation causes a novel syndrome of multisystemic smooth muscle dysfunction. American journal of medical genetics Part A. 2010;152A:2437-43.

[17] Wang L, Guo DC, Cao J, et al. Mutations in myosin light chain kinase cause familial aortic dissections. American Journal of Human Genetics. 2010;87:701-7.

[18] van de Laar IM, Oldenburg RA, Pals G, et al. Mutations in SMAD3 cause a syndromic form of aortic aneurysms and dissections with early-onset osteoarthritis. Nature genetics. 2011;43:121-6. 
[19] Loeys BL, Dietz HC, Braverman AC, et al. The revised Ghent nosology for the Marfan syndrome. Journal of Medical Genetics. 2010;47:476-85.

[20] Otterbein LR, Graceffa P, Dominguez R. The crystal structure of uncomplexed actin in the ADP state. Science. 2001;293:708-11.

[21] Roder C, Peters V, Kasuya H, et al. Analysis of ACTA2 in European Moyamoya disease patients. Eur J Paediatr Neurol. 2011;15:117-22.

[22] Owens GK. Regulation of differentiation of vascular smooth muscle cells. Physiological reviews. 1995; 75:487-517.

[23] Loeys BL, Schwarze U, Holm T, et al. Aneurysm syndromes caused by mutations in the TGF-beta receptor. N Engl J Med. 2006;355:788-98.

[24] Neptune ER, Frischmeyer PA, Arking DE, et al. Dysregulation of TGF-beta activation contributes to pathogenesis in Marfan syndrome. Nat Genet. 2003;33:407-11.

[25] Renard M, Holm T, Veith R, et al. Altered TGFbeta signaling and cardiovascular manifestations in patients with autosomal recessive cutis laxa type I caused by fibulin- 4 deficiency. European journal of human genetics : EJHG. 2010;18:895-901.

[26] Callewaert B, Renard M, Hucthagowder V, et al. New insights into the pathogenesis of autosomal-dominant cutis laxa with report of five ELN mutations. Human Mutation. 2011. [27] Lin F, Yang X. TGF-beta signaling in aortic aneurysm: another round of controversy. J Genet Genomics. 2010;37:583-91.

[28] Singh P, Carraher C, Schwarzbauer JE. Assembly of fibronectin extracellular matrix. Annu Rev Cell Dev Biol. 2010;26:397-419.

[29] Sabatier L, Chen D, Fagotto-Kaufmann C, et al. Fibrillin assembly requires fibronectin. Molecular biology of the cell. 2009;20:846-58. 
[30] Chen Q, Sivakumar P, Barley C, et al. Potential role for heparan sulfate proteoglycans in regulation of transforming growth factor-beta (TGF-beta) by modulating assembly of latent TGF-beta-binding protein-1. The Journal of biological chemistry. 2007;282:26418-30.

[31] Dallas SL, Sivakumar P, Jones CJ, et al. Fibronectin regulates latent transforming growth factor-beta (TGF beta) by controlling matrix assembly of latent TGF beta-binding protein- 1 . The Journal of biological chemistry. 2005;280:18871-80.

[32] Kantola AK, Keski-Oja J, Koli K. Fibronectin and heparin binding domains of latent TGF-beta binding protein (LTBP)-4 mediate matrix targeting and cell adhesion. Exp Cell Res. 2008;314:2488-500.

[33] Sivakumar P, Czirok A, Rongish BJ, Divakara VP, Wang YP, Dallas SL. New insights into extracellular matrix assembly and reorganization from dynamic imaging of extracellular matrix proteins in living osteoblasts. Journal of cell science. 2006;119:1350-60.

[34] Habashi JP, Judge DP, Holm TM, et al. Losartan, an AT1 antagonist, prevents aortic aneurysm in a mouse model of Marfan syndrome. Science. 2006;312:117-21.

[35] Shewan LG and Coats AJ. Ethics in the authorship and publishing of scientific articles. Int J Cardiol 2010;144:1-2. 


\section{Legends to figures}

\section{Figure 1}

Schematic overview of the ACTA2 (A,B) and MYH11 (C) protein. A. ACTA2 mutations previously described in the literature are marked in grey and the mutations we detected here are indicated in black. The numbers 1-9 indicate the exons. B. The ACTA2 mutations identified here (black) are mapped onto the crystal structure of actin (Protein Data Bank ID 1LVT). C. MYH11 mutations previously described in literature are indicated in grey and the mutation described in this article is indicated in black. The grey amino terminal part of the schematic protein represents the myosin head-like domain with 1 ATP binding site and 2 predicted actin binding sites, the black domain is the coiled-coil domain of the myosin heavy chain 11 protein.

\section{Figure 2}

Pedigrees of 7 families with ACTA2 mutation (family 1-7) and 1 family with MYH11 mutation (family 8). Legend: black box $=\mathrm{TAAD}$, arrow $=$ proband,$+=$ mutation present, $-=$ mutation absent.

\section{Figure 3}

Immunohistochemical staining of aortic tissue in ACTA2 (c-f) and MYH11 (g-h) mutationpositive patients and controls (a-b). Increased cytoplasmatic staining of CTGF and nuclear phospho-Smad2 accumulation is seen in the aortic tissue of patients with ACTA2 missense mutation (c and d, respectively) and MYH11 mutation ( $\mathrm{g}$ and $\mathrm{h}$, respectively) as compared to the control ( $\mathrm{a}$ and $\mathrm{b}$, respectively). No increase in CTGF nor pSmad2 staining is seen in the aortic tissue of a patient with ACTA2 nonsense mutation (e and f, respectively). 


\section{Figure 4}

Immunofluorescent staining of aortic tissue in ACTA2 (e-1) and MYH11 (m-p) mutationpositive patients and controls (a-d). Fragmented elastic fibers (FN1 - green) are seen in all patients $(\mathrm{g}, \mathrm{k}, \mathrm{o})$ as compared to the control (c). Smooth muscle $\alpha$-actin staining (red) is variable in ACTA2 mutation-positive patients (f,j) but seems to be increased in MYH11 mutation-positive patients (n) in comparison to the control (b). Smooth muscle cells in the control tissues (b) had an elongated shape (indicated with an arrow), while aortic tissue from patients $(f, j, n)$ showed the presence of few round shaped cells (indicated with an arrowhead).

\section{Figure 5}

Hypothetical model of the pathogenetic mechanism of MYH11 and ACTA2 mutations. A. Situation in healthy individuals: fibronectin assembly is initiated with the binding of fibronectin dimers onto an integrin receptor and syndecan, which possibly acts as a coreceptor. Integrins and syndecan are also involved in assembly of the actin cytoskeleton. Fibronectin fibrillogenesis is promoted by the integrin/cytoskeleton complex by cell contractility, which induces conformational changes in fibronectin, allowing the association of multiple fibronectin molecules and formation of fibrils. Fibrillin-1 C-terminal association and formation of bead-like structures depends on these fibronectin fibrils. Fibronectin fibrils are also required for the sequestration of latent TGF $\beta$, which is subsequently transferred onto the fibrillin-1 microfibrils, which are part of the elastic fibers. B. Mutations in MYH11 or ACTA2 will result in an incorrect assembly of the actin or myosin filaments, leading to a defect in fibronectin fibrillogenesis and resulting in an incorrect fibrillin-1 assembly into microfibrils and loss of the ability to sequestrate latent TGF $\beta$. Latent TGF $\beta$ that is not incorporated into the matrix is more prone to activation and can bind to its receptors, initiating TGF $\beta$ signaling, characterized by phosphorylation of the receptor Smads (R-Smad), binding 
to the Co-Smad. This complex is then translocated to the nucleus and together with coregulators can initiate the transcription of target genes. Used abbreviations: TGF $\beta R 1$ (Transforming growth factor $\beta$ receptor 1), TGF $\beta$ R2 (Transforming growth factor $\beta$ receptor 2), R-Smad (Receptor Smad), FN (fibronectin), FBN1 (fibrillin-1), LLC (large latent complex = LTBP (latent TGF $\beta$ binding protein) + LAP (latency associated peptide) + TGF $\beta$ ), SLC (small latent complex $=\mathrm{LAP}+\mathrm{TGF} \beta)$, TGF $\beta$ (Transforming growth factor $\beta)$. 